\title{
A SIMPLE PROOF OF ARAZY'S THEOREM
}

\author{
by J. A. ERDOS
}

(Received 23rd July 1992)

\begin{abstract}
Arazy has characterized the isometries of $\mathscr{C}_{p},(0<p \leqq \infty, p \neq 2)$ onto itself as all maps of the form $X \mapsto U X V$ where $U$ and $V$ are either both unitary or both anti-unitary. A simple proof of this result is given.

1991 Mathematics subject classification: 47B10, 47D25.
\end{abstract}

A new proof of the following characterization of the isometries of $\mathscr{C}_{p}$ onto itself is presented.

Arazy's theorem. Let $\phi$ be a linear isometry of $\mathscr{C}_{p}$ onto itself $(0<p \leqq \infty, p \neq 2)$. Then either

(i) $\phi(X)=U X V$ for some unitary operators $U$ and $V$ on the underlying Hilbert space, or

(ii) $\phi(X)=S X^{*} T$ for some anti-unitary operators $S$ and $T$ on the underlying Hilbert space.

The above result is proved in [1]. (See also [5]). The present proof shows clearly why the two cases arise and why $p=2$ is exceptional. An earlier version of this paper influenced a study of isometries of the intersections of nest algebras with $\mathscr{C}_{p}$ [2].

Here $\mathscr{C}_{p}$ denotes the von Neumann-Schatten $p$-class and anti-unitary operator means a conjugate-linear isometry on the Hilbert space onto itself. The underlying Hilbert space will be denoted by $\mathscr{H}$ and $\mathscr{R}$ and $\mathscr{F}$ will denote the rank 1 and finite rank operators on $\mathscr{H}$ respectively. The rank 1 operator $x \mapsto\langle x, e\rangle f$ will be written as $e \otimes f$. We shall repeatedly use the following elementary fact: if the sum of two rank one operators has rank one, the summands have either the same range or the same co-range.

The proof is based on two lemmas.

Lemma 1. Let $\phi$ be a linear map from $\mathscr{F}$ to $\mathscr{F}$ which preserves rank and is isometric on $\mathscr{R}$ (with respect to the operator norm). Then $\phi$ has one of the forms (i) or (ii) in the statement of Arazy's theorem.

Lemma 2. If $0<p<\infty$ and $p \neq 2$ then every linear isometry of $\mathscr{C}_{p}$ onto itself preserves rank. 
Arazy's theorem is an immediate consequence; one simply notes that the operator norm and the $\mathscr{C}_{p}$ norm (or metric when $0<p<1$ ) coincide on $\mathscr{R}$ and that $\mathscr{R}$ is dense in $\mathscr{C}_{p}$. The case $p=\infty$ follows from the case $p=1$ by a simple duality argument.

Proof of Lemma 1. Let $e$ be any unit vector. Then $\phi(e \otimes e)$ can be written as $f \otimes g$ with $\|f\|=\|g\|=1$. For any $x$ in $H, f \otimes g+\phi(e \otimes x)=\phi(e \otimes(e+x)) \in \mathscr{R}$ and hence either

$$
\phi(e \otimes x)=f \otimes \xi_{x} \quad \text { for some } \xi_{x} \in H
$$

or

$$
\phi(e \otimes x)=\eta_{x} \otimes g \text { for some } \eta_{x} \in H
$$

Either (1) or (2) must hold simultaneously for all vectors since, if (2) is false for $x$ and (1) is false for $y$ then both $\left\{\xi_{x}, g\right\}$ and $\left\{f, \eta_{y}\right\}$ are linearly independent pairs of vectors. This is impossible, since $f \otimes \xi_{x}+\eta_{y} \otimes g=\phi(e \otimes x+e \otimes y)$ has rank 1 .

Suppose (1) holds for all $x$. We show that the map $x \mapsto \xi_{x}$ is a linear isometry of $H$ onto $H$. Clearly $\|x\|=\|e \otimes x\|=\left\|f \otimes \xi_{x}\right\|=\left\|\xi_{x}\right\|$. Also, since $\phi(\mathscr{R})=\mathscr{R}$, for each $h \in H$, $f \otimes h=\phi(p \otimes q)$ for some $p, q \in H$. Now $f \otimes\left(\xi_{x}+h\right)=\phi(e \otimes x+p \otimes q)$ and so $e \otimes x+$ $p \otimes q \in \mathscr{R}$ for all $x$. Hence $p$ is a scalar multiple of $e$ and so $h=\xi_{t}$ where $t$ is some multiple of $q$. Therefore

$$
\phi(e \otimes x)=f \otimes U x \text { for some unitary operator } U
$$

If (2) holds for all $x$, then $\phi(e \otimes \lambda x)=\lambda\left(\xi_{x} \otimes g\right)=\left(\lambda \xi_{x}\right) \otimes g$, and so $x \mapsto \xi_{x}$ is conjugate linear. It now follows exactly as above that for some anti-unitary operator $W$, $\phi(e \otimes x)=W x \otimes g$. Since $W^{*}$ is also anti-unitary (adjoint being defined in the obvious way), we may write this as

$$
\phi(e \otimes x)=T^{*} x \otimes g \text { for some anti-unitary operator } T .
$$

Now consider $\phi(y \otimes e)$ as $y$ varies. It follows that either

$$
\phi(y \otimes e)=V^{*} y \otimes g \text { for some unitary operator } V .
$$

or

$$
\phi(y \otimes e)=f \otimes S^{*} y \quad \text { for some anti-unitary operator } U
$$

Conditions (1a) and (2b) cannot hold simultaneously since this would imply that $e \otimes x+y \otimes e$ has rank 1 for all $x$ and $y$. Similarly (1b) and (2a) are incompatible.

Suppose that (1a) and (1b) hold. If $\phi(s \otimes t)=v \otimes u$ and neither $s$ nor $t$ is a multiple of $e$, then $\phi(e \otimes e+s \otimes t) \notin \mathscr{R}$ and $\phi(e \otimes t+s \otimes t) \in \mathscr{R}$. That is, $f \otimes g+v \otimes u \notin \mathscr{R}$ and $f \otimes U t+v \otimes u \in \mathscr{R}$. Therefore $u$ is a multiple of $U t$. Similarly $v$ is a multiple of $V^{*} s$. It follows that for arbitrary $s, t$ 


$$
\phi(s \otimes t)=\mu(s, t)\left(V^{*} s \otimes U_{t}\right)
$$

where $\mu(s, t)$ is some complex number, possibly depending on $s$ and $t$. But, for fixed $s$, $\phi(s \otimes t)$ is linear in $t$, so

$$
\mu\left(s, t_{1}+t_{2}\right)\left[V^{*} s \otimes U\left(t_{1}+t_{2}\right)\right]=\mu\left(s, t_{1}\right)\left[V^{*} s \otimes U t_{1}\right]+\mu\left(s, t_{2}\right)\left[V^{*} s \otimes U t_{2}\right] .
$$

From the case when $t_{1}$ and $t_{2}$ are independent, it follows that $\mu\left(s, t_{1}\right)=\mu\left(s, t_{1}+t_{2}\right)=$ $\mu\left(s, t_{2}\right)$ and so $\mu$ is independent of $t$. Similarly $\mu$ is independent of $s$ and so $\mu$ is constant. Since $\mu(e, e)=1$, we have

$$
\phi(s \otimes t)=V^{*} s \otimes U t=U(s \otimes t) V
$$

and $\phi$ satisfies (i). If (2a) and (2b) hold, it follows in the same way that $\phi$ satisfies (ii).

Proof of Lemma 2. It is clearly sufficient to show that $\phi(\mathscr{R}) \in \mathscr{R}$ for each $\mathscr{R} \in \mathscr{R}$. We use the conditions for equality in the Clarkson-McCarthy inequality. The result is: for $X, Y \in \mathscr{C}_{p},(0<p<\infty, p \neq 2)$,

$$
\|X+Y\|_{p}^{p}+\|X-Y\|_{p}^{p}=2\left(\|X\|_{p}^{p}+\|Y\|_{p}^{p}\right)
$$

if and only if $Y^{*} X=Y X^{*}=0$ (see [4]). Note that for $p=2$ equality always holds. For the present purpose, we only need the observation that, since the condition is that $\operatorname{ran}(X) \perp \operatorname{ran}(Y)$ and $\operatorname{ran}\left(X^{*}\right) \perp \operatorname{ran}\left(Y^{*}\right)$, equality cannot hold if $X+Y$ has rank 1 (unless $X=0$ or $Y=0$ ).

Suppose $A$ has rank 1 and $\phi(A)=T$ has rank $>1$. Using the standard Schmidt decomposition (see e.g. [3]), $T=\Sigma \mu_{i}\left(x_{i} \otimes y_{i}\right)$ where $\left\{x_{i}\right\}$ and $\left\{y_{i}\right\}$ are orthonormal. Thus, $T=X+Y$ where $X=\mu_{1}\left(x_{1} \otimes y_{1}\right) \neq 0$ and $Y=\Sigma_{i>1} \mu_{i}\left(x_{i} \otimes y_{i}\right) \neq 0$. It follows that

$$
\|X \pm Y\|_{p}^{p}=\Sigma \mu_{i}^{p}=\|X\|_{p}^{p}+\|Y\|_{p}^{p}
$$

and so $\left(^{*}\right)$ holds. Applying the isometric transformation inverse to $\phi$ gives a similar decomposition of $A$, and this contradiction shows that $\phi(A)$ has rank 1 , thus completing the proof.

Remarks. 1. In [1], case (ii) of the Theorem is stated as:

(ii)' $\phi(X)=U X^{T} V$ for some unitary operators $U$ and $V$.

where $X^{T}$ is the transpose of $X$ with respect to some orthonormal basis $\left\{e_{i}\right\}$ of the underlying Hilbert space.

To see that (ii) and (ii)' are the same, consider the conjugation $C: H \mapsto H$ by

$$
C x=\sum_{i} \overline{\left\langle x, e_{i}\right\rangle} e_{i} .
$$


Clearly $C^{2}=C$ and $C$ is anti-unitary. Thus if $X^{T}$ is the transpose of $X$ with respect to the basis $\left\{e_{i}\right\}$, then $C X^{T} C=X^{*}$ and, if $U$ and $V$ are anti-unitary, $U C$ and $C V$ are anti-unitary. The equivalence now follows.

2. Arazy's original proof also uses the condition for equality in the ClarksonMcCarthy inequality to show that rank is preserved. However, the argument is more involved.

3. The result is also proved by Sourour [5] using quite different methods which also cover more general symmetrically normed ideals.

\section{REFERENCES}

1. J. Arazy, The isometries of $\mathscr{C}_{p}$, Israel J. Math. 22 (1975), 247-256.

2. M. Anoussis and A. Katavolos, $\mathscr{C}_{p}$ isometries of some operator algebras, preprint.

3. I. C. Gohberg and M. G. Krein, Theory and Applications of Volterra Operators in Hilbert Space (Transl. Math. Monographs 24, Amer. Math. Soc., Providence, R.I., 1970).

4. C. A. McСаrthy, $\mathscr{C}_{p}$, Israel J. Math. 5 (1967), 249-271.

5. A. R. Sourour, Isometries of norm ideals of compact operators, J. Funct. Anal. 43 (1981), 69-77.

Department of Mathematics

King's College

LONDON WC2R 2LS 